\section{In Vitro Seed Germination of Kalmia latifolia L. Hybrids: A Means for Improving Germination and Speeding Up Breeding Cycle}

\author{
He Li and Donglin Zhang ${ }^{1}$ \\ Department of Horticulture, University of Georgia, 1111 Miller Plant \\ Sciences Building, Athens, GA 30602
}

Additional index words. ericaceous, mountain laurel, ornamental plants, rapid breeding, tissue culture

\begin{abstract}
Kalmia latifolia L. (mountain laurel), a member of Ericaceae, is a beautiful ornamental shrub native to the eastern United States. The plant is not common in the southeastern United States landscapes because of the limited heat tolerance of most commercial cultivars. Breeding of heat-tolerant cultivars can be achieved by cross hybridization, but is often challenged by low germination percentage, long germination time, and potential abortion of cross-hybridized seeds. We used in vitro seed germination to enhance germination and shorten germination time and investigated the appropriate collecting time, optimal basal medium, and $\mathrm{pH}$ for this approach. Collecting time affected in vitro seed germination, with more mature hybrid seeds [collected 4-5 months after pollination (MAP)] having higher germination rate $(90 \%$ in 4 weeks) than the less mature seeds collected in 2 MAP (20\% in 7 weeks). Seedlings from the mature seeds also produced two true leaves on average after 8 weeks of culture, whereas seedlings from the less mature seeds had no true leaves. Woody Plant Medium (WPM) better enhanced in vitro seed germination compared with Murashige and Skoog (MS) or Gamborg's B5 (B5) medium. WPM yielded higher germination $(98 \%)$ than MS $(90 \%)$ and significantly greater total leaf area per seedling $\left(67 \mathrm{~mm}^{3}\right)$ than MS $\left(50 \mathrm{~mm}^{3}\right)$ and B5 $\left(52 \mathrm{~mm}^{3}\right)$ for seeds of 'Firecracker' $x$ 'Snowdrift'. Similar effects had been observed on seeds from 'Little Linda' $\times$ 'Starburst' and 'Pristine' $\times$ 'Peppermint'. The pH ranging from 4.2 to 5.4 did not affect seed germination and seedling development of mountain laurel hybrids. Our protocol enabled early collection of mountain laurel hybrid seeds 1 month before their full maturation and permitted seeds to germinate in 4 weeks on WPM, which shortened the period from crossing to the seedling stage from up to 15 to 6 months and enhanced germination percentage from $30 \%$ to more than $90 \%$ compared with traditional seed germination. This protocol should be applied to promote the breeding and selection of new mountain laurel cultivars for the southeastern United States landscapes.
\end{abstract}

Kalmia latifolia L. (mountain laurel), a member of Ericaceae (heath family), is a large evergreen flowering shrub native to the eastern United States. It has been considered by many horticulturists and gardeners to be one of the most beautiful flowering species because of its attractive lustrous green foliage and showy inflorescence (Dirr, 2011; Jaynes, 1988). It usually blooms in late spring or early summer on new shoots. The inflorescence consists of a terminal compound corymb with numerous showy flowers. The variations in flower shape and color, leaf shape and size, and habit have made mountain laurel a valuable ornamental shrub in gardens and landscapes (Jaynes, 1988). More than 100 mountain laurel cultivars have been released through the effort of ornamental

Received for publication 29 Dec. 2017. Accepted for publication $1 \mathrm{Feb} .2018$.

We highly appreciate the support from the University of Georgia Research Foundation (Project Number: 2521RC297295).

${ }^{1}$ Corresponding author. E-mail: donglin@uga.edu. and uneven germination and long germination period thus slow down the breeding cycle. Furthermore, seeds from cross hybridization may abort because of the incompatibility. A better and faster method to germinate seeds of mountain laurel hybrids is needed to speed up breeding of new cultivars

In vitro culture is the cultivation of plant tissues or organs under aseptic conditions in an artificial medium of known chemical composition. For the last 50 years, this culture technique has been widely used on many plants to obtain seedlings from interspecific and intergeneric breeding, of which embryos lack of endosperm and seeds fail to germinate using conventional methods (Bridgen, 1994; De Jeu, 2000; Sharma, 1995). By culturing immature embryos or seeds in the nutrient medium, some can overcome the incompatibility and avoid abortion. Successful case has been documented for another member of heath familyrhododendron hybrids (Eeckhaut et al., 2007). Some types of seeds only germinate after a period of storage to overcome dormancy, and in vitro culture can break seed dormancy and shorten seed germination time (Sharma et al., 1996). Therefore, in vitro seed germination methods would be a potential means to enhance seed germination of mountain laurel.

Successful in vitro culture depends on many factors and the protocol varies depending on genotypes. Medium selection is considered as one of the most important factors for tissue culture. Several formulations such as MS (Murashige and Skoog, 1962), B5 medium (Gamborg et al., 1968), and WPM (Lloyd and McCown, 1980) with certain degrees of modification, are the most widely used basal media in embryo and seed culture (Askari-Khorasgani et al., 2013; Hao et al., 2014; Pavlovic et al., 2012). The $\mathrm{pH}$ of media can also affect plant growth (Reeves et al., 1983). Generally, the pH of tissue culture media is maintained around 5.7-5.9, whereas adjusted $\mathrm{pH}$ can improve the embryo development and plant growth for some species (Ribeiro et al., 1999; Zhang et al., 2004). In addition to these cultivation conditions, determining the appropriate time to collect seeds is a key factor for the success of in vitro culture. Seeds may fail to germinate when they are harvested in their early stage whereas later collection could lead to increasing contamination rates (Yang et al., 2015).

In this study, we investigated the effects of seed maturity, basal medium, and $\mathrm{pH}$ on the germination percentage and speed, as well as seedling vigor for several mountain laurel hybrids. Our objective was to establish an efficient in vitro seed germination protocol for mountain laurel hybrid seeds to enable seed harvest before full maturity, shorten the time required for germination by breaking down dormancy, and improve seed germination by providing favorable conditions. This protocol would be an approach to speed up the breeding cycle and promote the breeding 
and selection of new mountain laurel cultivars for the southeastern landscapes.

\section{Materials and Methods}

Plant materials, sterilization, and culture conditions. All fruit capsules used in the study were obtained from artificial crosses between mountain laurel cultivars. Female parents were emasculated and hand pollinated in early May 2016 under greenhouse conditions at the University of Georgia Horticulture Farm (Watkinsville, GA). Capsules were harvested from mother plants and washed with running tap water for $30 \mathrm{~min}$. Capsules were then surface disinfected with $50 \%$ Clorox solution $(4.15 \%$ sodium hypochlorite; The Clorox Company, Oakland, CA) added with one drop of Tween 20 (Hoefer, Holliston, MA) for $6 \mathrm{~min}$, and subsequently rinsed four times with autoclaved distilled water in a laminar flow hood. The surface-sterilized capsules were kept in sterile water until dissection. Seeds were extracted under a dissecting microscope (Fisher Scientific Education, Nazareth, PA) and isolated seeds were transferred onto the surface of medium in $6-\mathrm{cm}$ petri dishes. Each petri dish contained $10 \mathrm{~mL}$ of basal media supplemented with 3\% sucrose (Sigma-Aldrich, St. Louis, MO) and $0.6 \%$ agar (Fisher Science Education) and was sealed with parafilm (Bemis Company, Oshkosh, WI) after sowing. Eight weeks after culture, measurements were taken and seedlings were subcultured to $10-\mathrm{cm}$ petri dishes filled with $25 \mathrm{~mL}$ of same basal medium. Subculture was then made every 4 weeks. All petri dishes were incubated in a culture room at $25 \pm 2{ }^{\circ} \mathrm{C}$ under cool-white fluorescent lamps [photosynthetic photon flux $(P P F)$ of $66 \mu \mathrm{mol} \cdot \mathrm{m}^{-2} \cdot \mathrm{s}^{-1}$ for $14-\mathrm{h}$ photoperiod because light is required for germination of mountain laurel seeds (Malek et al., 1989).

Effect of seed-collecting time on germination. Fruit capsules from the crosses 'Elf' $\times$ 'Little Linda' and 'Red Bandit' $\times$ 'Minuet' were harvested every month from July to Oct. 2016 (2-5 MAP). Capsule growth was measured by a ruler and the appearance was photographed. Extracted seeds were observed and photographed under dissecting microscope. Culture medium contained B5 basal medium supplemented with $3 \%$ sucrose and $0.6 \%$ agar, and $\mathrm{pH}$ was adjusted to 5.0. All cultures were maintained under the aforementioned environmental conditions.

Effect of basal medium on seed germination. Hybrid capsules from 'Little Linda' $\times$ 'Starburst', 'Firecracker' $\times$ 'Snowdrift', and 'Pristine' $\times$ 'Peppermint' were collected on 15 Sept. 2016. The surfacedisinfected capsules were dissected and seeds were isolated from capsules under a microscope. The isolated seeds were cultured in $6-\mathrm{cm}$ petri dishes containing either 1) MS, 2) WPM, or 3) B5 basal medium plus 3\% sucrose and $0.6 \%$ agar. The $\mathrm{pH}$ of media was adjusted to 5.0 before adding agar.
Effect of $p H$ on seed germination. Hybrid capsules from 'Little Linda' $\times$ 'Starburst', 'Firecracker' $\times$ 'Snowdrift', and 'Pristine' $\times$ 'Peppermint' were harvested on 16 Sept. 2016. Capsules were disinfected following the aforementioned procedure. The extracted seeds were sowed into B5 medium with following $\mathrm{pH}:$ 1) 4.2, 2) 4.6, 3) 5.0, or 4) 5.4. All petri dishes were incubated in the culture room as previously mentioned.

Acclimatization. After two cycles of subcultures, more than 300 vigorous seedlings (with at least four true leaves) of all crosses were taken out from petri dishes and washed with distilled water to remove remains of agar. Seedlings were then transplanted to presoaked peatmoss in 32-cell flat trays for acclimatization and subsequent growth. Trays were covered with plastic dome to maintain high humidity. The dome was gradually vented after seedlings started to harden off and was eventually removed during acclimatization. All plants were kept in a growth chamber at $25 \pm 2{ }^{\circ} \mathrm{C}$ under a 16 -h photoperiod provided by cool-white fluorescent lamps at $P P F$ of $66 \mu \mathrm{mol} \cdot \mathrm{m}^{-2} \cdot \mathrm{s}^{-1}$. Seedlings were fertilized with $50 \mathrm{ppm} 15 \mathrm{~N}-4 \mathrm{P}-15 \mathrm{~K}$ water-soluble fertilizer (J.R. Peters, Inc., Allentown, PA) weekly and watered as needed.

Experimental design and data analysis. All studies were performed in a completely randomized design with four replications (petri dishes) per treatment and 10 seeds per treatment per replication. Seed germination was observed weekly under microscope and visible radicle emergence was counted as germination (Fig. 1C). Germination percentage was calculated as (number of seeds showing radicle emergence 8 weeks after culture/total number of seeds cultured) $\times$ $100 \%$. Germination speed (T100) was calculated as the number of weeks reaching $100 \%$ of total germination. Individual petri dishes were photographed 8 weeks after culture for seedling growth analysis. Number of true leaf and total leaf area were measured using ImageJ 1.x (Fig. 1D; Schneider et al., 2012). Data were analyzed by analysis of variance and GLM program in SAS University Edition (SAS Institute, Cary, NC) and the significance level was set at $P<0.05$.

\section{Results and Discussion}

The effect of collecting time on capsule development and seed germination. Capsules were successfully harvested from both crosses ('Elf' $\times$ 'Little Linda' and 'Red Bandit' $\times$ 'Minuet') 2-5 MAP. Capsule expansion completed in the first 2 months with a significant increase in capsule diameter and no further growth thereafter. Capsule color changed from green to yellow and then to brown during collecting period, with the capsule of 'Red Bandit' $\times$ 'Minuet' ripened earlier than that of 'Elf' $\times$ 'Little Linda'. The capsule from the cross between 'Elf' and 'Little Linda' was smaller compared with that from 'Red Bandit' $\times$ 'Minuet' at the same collecting time (Fig. 1A). This is because that the female parent 'Elf' is a miniature cultivar with a dwarf growth habit and reduced leaf and flower size and therefore produces smaller fruit than nondwarf cultivar 'Red Bandit'. Seeds from both crosses were small, ranging from 0.7 to $0.9 \mathrm{~mm}$ in length and 0.3 to $0.4 \mathrm{~mm}$ in width (Fig. 1B). This is the normal seed size of mountain laurel (Jaynes, 1988). Both crosses did not show significant changes in seed size or seed color from 2 to 5 MAP.

The germination capability of hybrid seeds of mountain laurel varied with the collecting time. Less than $20 \%$ of seeds germinated when the fruit capsule was collected in July (2 MAP), whereas germination percentage of August collection for cross 'Red Bandit' $\times$ 'Minuet' and cross 'Elf' $\times$ 'Little Linda' increased significantly to $65.0 \%$ and $92.5 \%$, respectively (Fig. $2 A$ ). In September and October collections, both crosses yielded more than $90.0 \%$ of germination. However, contamination percentage increased significantly in October collection, particularly for the hybrids between 'Red Bandit' $\times$ 'Minuet' $(12.5 \%)$ that ripened earlier than the other cross (Fig. 2B). Hybrid seeds from both crosses tended to have faster and more even germination when they were harvested later, indicated by a negative correlation between $\mathrm{T} 100$ and collecting time (Fig. 2C). T100 was 6.5 weeks in early collection for the cross 'Elf' $\times$ 'Little Linda' whereas T100 in September and October collections was reduced to $\approx 4$ weeks, although the difference was not significant. A similar trend was observed with the hybrid seeds from cross 'Red Bandit' $\times$ 'Minuet' (Fig. 2C). Germinated seedlings became increasingly vigorous as harvesting time went later. Seedlings from seeds that were harvested at the early stage ( 2 MAP) produced no true leaf after 8 weeks of culture, only radicles or cotyledons were observed. In contrast, seedlings were able to yield significantly more true leaves when seeds were collected in later collections, particularly October collection having two true leaves on average per seedling (Fig. 2D). Results showed that collecting time affected in vitro seed germination, with more mature hybrid seeds having higher capacity to germinate compared with immature seeds. Similar results were reported on closely related ericaceous species rhododendron by Michishita et al. (2001) that less mature hybrid seeds had lower ability to germinate in vitro compared with mature seeds. Geng et al. (2014) also found that younger rhododendron hybrid embryos were more likely to only form callus while relatively mature embryos tended to germinate successfully into seedlings. Mountain laurel seeds generally start to ripen in 4 MAP and become fully mature in 5 MAP, although maturation time varies a bit from year to year and among plants. Two-monthold capsules did not yield any seedlings because of the underdeveloped embryos at this stage, whereas most of the seeds harvested in September (4 MAP) were able to germinate evenly because seeds became mature and embryos enabled the radicle to 


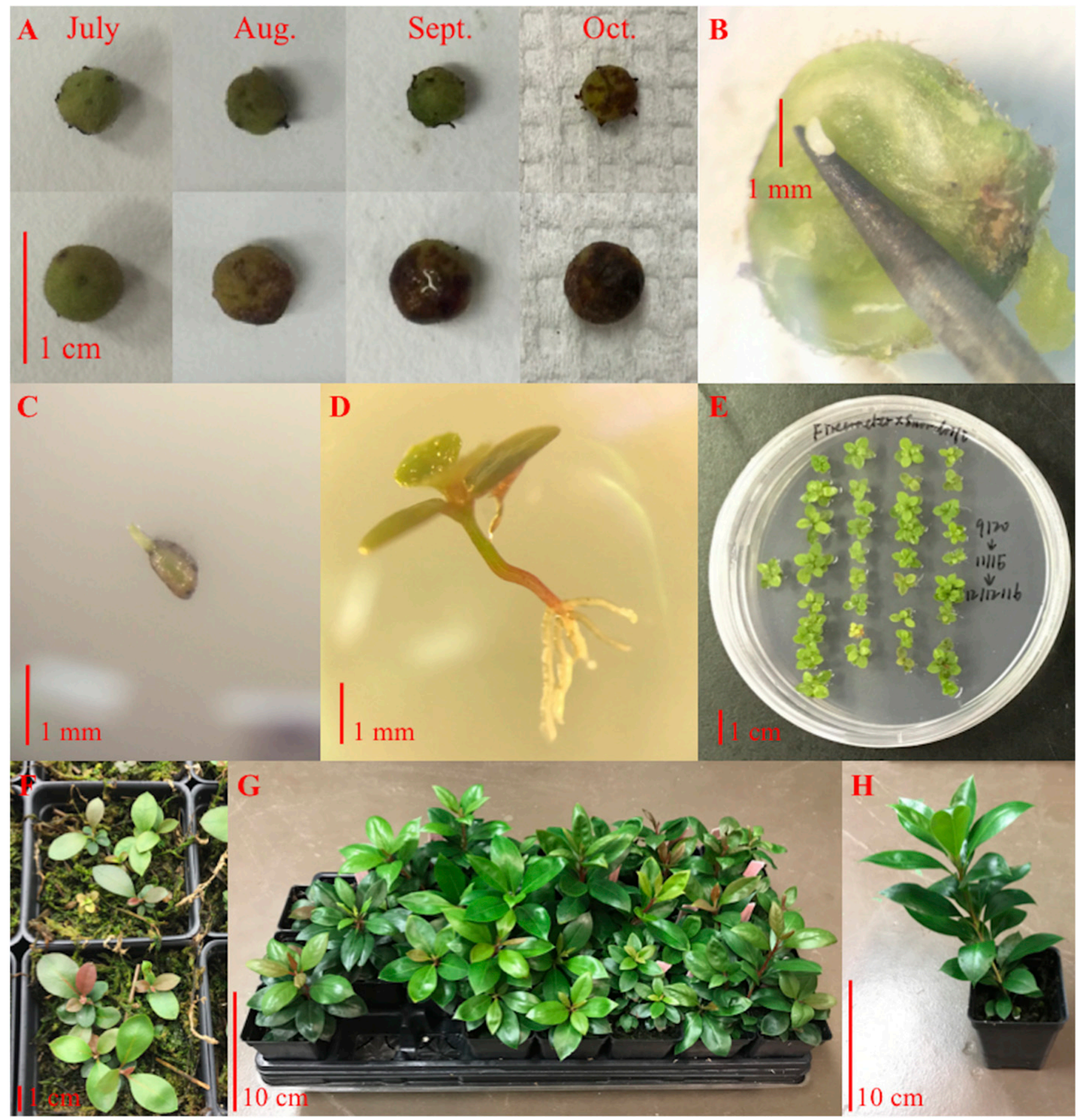

Fig. 1. In vitro seed germination of mountain laurel hybrids. (A) Capsule development during the collecting time (from July to October) of crosses 'Elf' $\times$ 'Little Linda' (top) and 'Red Bandit' $\times$ 'Minuet' (bottom). (B) Seed extracted from the disinfected capsule in the laminar flow hood, which would then be sowed on medium in 6-cm petri dish. (C) Seed germinated in 2 weeks after culture as radicle emergence was observed. (D) Eight weeks after culture, seedlings were photographed for data collection and then subcultivated to fresh medium. (E) Seedlings in 10-cm petri dish after two cycles of subcultures; vigorous ones would be transplanted to presoaked peatmoss for acclimatization and further development. (F) Seedling growth after being acclimatized in the growth chamber for 3 weeks. And ( $\mathbf{G}$ and $\mathbf{H}$ ) after 3-month acclimatization, seedlings were hardened off and yielded significant shoot growth.

penetrate the endosperm and seedcoat (Jaynes, 1988; Taiz and Zeiger, 2010). Later collection thus yielded higher germination percentages, more leaf growth, and faster germination than early collection. However, capsules might start to dehisce in 5 MAP and the longer the capsules were exposed to field conditions, the higher the infection occurred, which led to increased contamination rates in
October collection. Four MAP therefore would be recommended to be the appropriate time for collecting seeds for in vitro culture. The seeds of 'Red Bandit' $\times$ 'Minuet' had overall lower germination ability, including germination percentage and seedling vigor, compared with that of 'Elf' $\times$ 'Little Linda' (Fig. 2). This is probably because mountain laurel is a cross-pollinated species and cultivars
'Red Bandit' and 'Minuet' had relatively higher genetic similarity $(72.73 \%)$ than that of the other cross $(69.09 \%)$ (results from a separate study) and their hybrid seeds areless capable to germinate because of higher inbreeding depression. The overall results showed that collecting and cultivating 4-month-old hybrid seeds of both crosses in vitro could shorten the period from crossing to 
the seedling stage from $13-15$ months to 6 months and improve germination percentage from $30 \%$ to more than $90 \%$, which is of significance for plant breeding programs.

Effect of basal medium on seed germination. The highest germination percentages were obtained with the treatment of WPM in 8 weeks for all three crosses (Table 1). The cross 'Little Linda' $\times$ 'Starburst' yielded significantly more germination on WPM $(90.0 \%)$ than that of MS $(72.5 \%)$ and B5 $(72.5 \%)$, whereas 'Firecracker' $\times$ 'Snowdrift' and 'Pristine' $\times$ 'Peppermint' had 97.5\% and $92.5 \%$ of germination, respectively, with both WPM and B5. Although WPM and B5 induced similar germination percentage, the smaller T100 of WPM (2.0-3.5 weeks) indicated that WPM tended to have higher capacity of shortening germination time compared with B5 (T100 of 2.8-4.5 weeks). In terms of seedling vigor, the results showed that the effect of basal medium on true leaf number per seedling was genotype-specific. MS promoted more leaf production of the seedlings of 'Little
Linda' $\times$ 'Starburst' and 'Firecracker' $\times$ 'Snowdrift' whereas WPM enhanced that of 'Pristine' $\times$ 'Peppermint', but the difference was not statistically significant. In contrast, there was a trend between total leaf area per seedling and medium types that WPM yielded larger leaf area than B5 and MS and the difference was significant with the cross 'Firecracker' $\times$ 'Snowdrift'. Foliage chlorosis was observed on seedlings in B5. The data showed that WPM better enhanced in vitro seed germination of
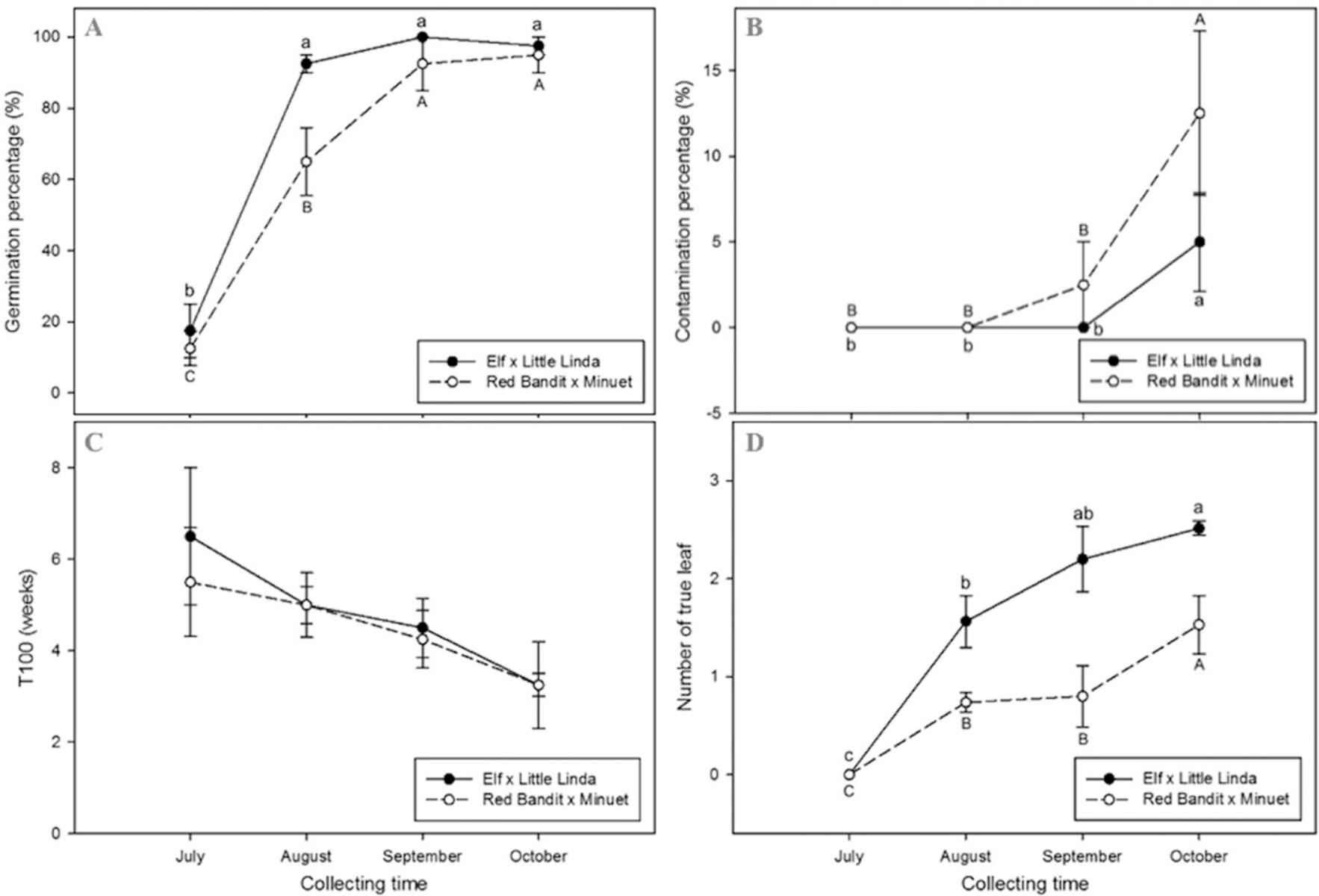

Fig. 2. Effect of collecting time on in vitro seed germination, including (A) germination percentage, (B) contamination percentage, $(\mathbf{C})$ T100, indicator for germination speed, and (D) number of true leaf per seedling, of mountain laurel hybrids 'Elf' $\times$ 'Little Linda' (solid line with closed circle) and 'Red Bandit' $\times$ 'Minuet' (dash line with open circle). Germination \%, contamination \%, and number of true leaf were measured 8 weeks after in vitro culture. T100 was calculated as the number of weeks reaching $100 \%$ of total germination. Data are present as mean of four replications \pm SE. Different letters within each cross combination indicate that they are significantly different at $P<0.05$.

Table 1. Effect of basal medium on in vitro germination of 4-MAP hybrid seeds of Kalmia latifolia.

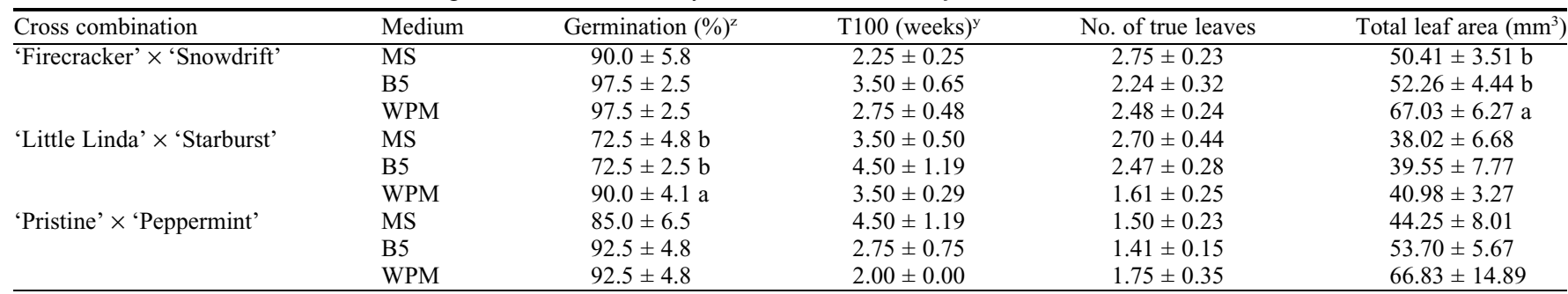

${ }^{\mathrm{z}}$ Data are present as mean of four replications \pm SE. Means followed by different letters within each cross combination in a column indicate that they are significantly different at $P<0.05$.

${ }^{\mathrm{y}}$ Seed germination was recorded weekly and T100 (indicating germination speed) was calculated as the number of weeks reaching $100 \%$ of total germination. MS = Murashige and Skoog; B5 = Gamborg's B5; WPM = woody plant medium; MAP = months after pollination. 
mountain laurel hybrids than MS and B5, which was consistent with the findings of Geng et al. (2014). In their study, WPM was found to be the optimal medium for seed germination of rhododendron hybrids that indicated by higher germination percentage and better seedling growth compared with other media including MS, 1/4MS, and Anderson (Anderson, 1980). WPM is originally designed for micropropagation of mountain laurel by Lloyd and McCown (1980) for the purpose of propagating mountain laurel clones in large scales to fulfill commercial demand. WPM has then been widely used on many other dicot ornamental species, such as rose, birch, and oak, for their tissue cultures (McCown, 2000). The major difference between WPM and MS is that MS has overall higher nutrient contents, particularly nitrogen ( $>4$-fold), than WPM (Lloyd and McCown, 1980; Murashige and Skoog, 1962). This high nutrient content of MS might be excessive to tiny young seeds and thus suppressed their germination and seedling development. In contrast, B5 has low inorganic nutrient levels, such as calcium and magnesium, which might lead to nutrient deficiency and inhibit seedling growth. In addition to the low nutrient levels, the primary nitrogen source of B5 is nitrate $\left(\mathrm{NO}_{3}{ }^{-}\right)$, which might account for the stunted growth as well. Many ericaceous members, including rhododendrons and blueberries, have a preference for the ammonium form $\left(\mathrm{NH}_{4}^{+}\right)$over the nitrate form $\left(\mathrm{NO}_{3}{ }^{-}\right)$of inorganic nitrogen. In the absence of this preferred condition, these ericaceous plants may display foliar chlorosis and stunted shoot growth (Alt et al., 2017; Clark et al., 2003). In B5 medium, the $\mathrm{NH}_{4}{ }^{+} / \mathrm{NO}_{3}{ }^{-}$ ratio is 0.08 , much lower than that of WPM and MS (0.52) (Gamborg et al., 1968). Therefore, the low nutrient level and $\mathrm{NO}_{3}{ }^{-}$ as the dominant nitrogen form of B5 medium probably limited seed germination and resulted in foliage chlorosis and smaller seedlings of mountain laurel hybrids. No significant difference in seed germination between cross combinations was observed; however, 'Little Linda' $\times$ 'Starburst' had a smaller total leaf area but similar leaf number than other two crosses. Because 'Little Linda' and 'Starburst' are miniature cultivars that produce smaller leaves than other normal cultivars and this trait is controlled by a single recessive gene (Jaynes, 1988), their progenies were all miniatures producing reduced leaf and had relatively smaller total leaf area.

Effect of $\mathrm{pH}$ on seed germination. $\mathrm{pH}$ values did not show significantly different effect on in vitro seed germination of three mountain laurel hybrids (Table 2). Seeds collected from all crosses ('Little Linda' $\times$ 'Starburst', 'Firecracker' $\times$ 'Snowdrift', and 'Pristine' $\times$ 'Peppermint') yielded more than $80 \%$ of germination in 4 weeks under all $\mathrm{pH}$ treatments. Neither number of true leaf nor total leaf area displayed significant difference between treatments within each cross combination. Like most ericaceous members such as rhododendrons and blueberries, mountain laurel is considered to prefer acidic soil condition ranging between 4.0 and 5.5 (Jaynes, 1988). However, our results showed that none of these hybrid seeds exhibited a $\mathrm{pH}$ preference by their in vitro germination. This could be due to that the $\mathrm{pH}$ range (4.2-5.4) used in our study fell into the desired $\mathrm{pH}$ conditions of mountain laurel, and thus, all treatments favored seed germination. Alternatively, the preference on acidic soil condition may not be attributed by $\mathrm{pH}$ per se but by the nutrient availability under low $\mathrm{pH}$ values. Under acidic soil conditions, $\mathrm{NH}_{4}{ }^{+}$is present as the primary form of inorganic nitrogen rather than $\mathrm{NO}_{3}^{-}$(Alt et al., 2017). As previously mentioned, many of ericaceous plants prefer $\mathrm{NH}_{4}{ }^{+}$over $\mathrm{NO}_{3}{ }^{-}$of inorganic nitrogen (Alt et al., 2017; Clark et al., 2003). This probably accounts for adaptation of heath family plants to the soil with low $\mathrm{pH}$. Availability of other nutrients, such as iron, may also cause this preference for low $\mathrm{pH}$. Under our tissue culture condition, the hybrid seeds were exposed to the same nutrient components when testing between different $\mathrm{pH}$ treatments. Therefore, $\mathrm{pH}$ did not significantly influence in vitro seed germination and seedlings vigor in our study. When comparing germination of three hybrid seeds, the similar trend was found as in basal medium study that seedlings from 'Little Linda' $\times$ 'Starburst' had smaller total leaf area than that of other two crosses because two parents involved in this cross are miniatures.
Acclimatization. Seedlings having at least four true leaves were transplanted into presoaked peatmoss in 32-cell flat trays for acclimatization and subsequent growth. Seedlings displayed significant aboveground growth in 3 weeks following transplanting (Fig. 1F). After 3-month acclimatization, $83.9 \%$ of seedlings survived and yielded well-developed shoot and root system (Fig. $1 \mathrm{G}$ and $\mathrm{H}$ ). Seedlings were subsequently transplanted to 1-gallon pots filled with pine bark as substrate for future evaluation.

\section{Conclusions}

Mountain laurel seeds are generally collected in 5-6 MAP when they are fully mature. Seeds will be then stored at $4{ }^{\circ} \mathrm{C}$ and sowed in the following spring using traditional seed germination. A mix of peatmoss and perlite at a ratio of 2:1 is used as substrate, and seeds should be sown on the surface of the mix and not covered. The moist substrate and high humidity are necessary for germination (Jaynes, 1971). This protocol requires careful handling but only yields low germination percentage and the germination could take up to 3 months. The subsequent seedling growth is generally slow, which takes $\approx 1$ year to reach a plant height of $10 \mathrm{~cm}$. While using our in vitro seed germination protocol, hybrid seeds could be collected 1 month before their full maturation and seeds were able to germinate in 1 month on WPM, which shortened the period from crossing to the seedling stage from 13-15 months to 6 months and improved germination percentage from $30 \%$ to more than $90 \%$ compared with the traditional method. After two cycles of subcultures, seedlings were vigorous enough to be transplanted from petri dish to peatmoss for acclimatization. Seedlings with both great shoot and root system were obtained in 3 months after acclimatization. The protocol developed in this study therefore enables to increase the number of hybrid seedlings and to speed up the breeding cycle by improving germination percentage, shortening germination time, and enhancing seedling growth. This protocol thus can be an approach to promote the breeding and selection of new mountain laurel cultivars for the southeastern United States landscapes.

Table 2. Effect of $\mathrm{pH}$ on germination and seedling vigor of 4-MAP Kalmia latifolia hybrid seeds cultivated in vitro.

\begin{tabular}{|c|c|c|c|c|c|}
\hline Cross combination & $\mathrm{pH}$ & Germination $(\%)^{z}$ & T100 (weeks) ${ }^{\mathrm{y}}$ & No. of true leaves & Total leaf area $\left(\mathrm{mm}^{3}\right)$ \\
\hline \multirow[t]{4}{*}{ 'Firecracker' $\times$ 'Snowdrift' } & 4.2 & $90.0 \pm 7.1$ & $2.75 \pm 0.25$ & $2.15 \pm 0.39$ & $48.28 \pm 5.75$ \\
\hline & 4.6 & $92.5 \pm 4.8$ & $2.50 \pm 0.29$ & $2.20 \pm 0.12$ & $52.78 \pm 3.98$ \\
\hline & 5.0 & $95.0 \pm 2.9$ & $2.75 \pm 0.25$ & $2.32 \pm 0.28$ & $44.38 \pm 5.31$ \\
\hline & 5.4 & $95.0 \pm 2.9$ & $2.50 \pm 0.29$ & $2.28 \pm 0.21$ & $49.72 \pm 2.52$ \\
\hline \multirow[t]{4}{*}{ 'Little Linda' $\times$ 'Starburst' } & 4.2 & $80.0 \pm 5.8$ & $3.25 \pm 0.48$ & $2.33 \pm 0.41$ & $37.60 \pm 5.12$ \\
\hline & 4.6 & $77.5 \pm 10.3$ & $3.75 \pm 0.85$ & $1.98 \pm 0.15$ & $38.43 \pm 3.16$ \\
\hline & 5.0 & $82.5 \pm 4.8$ & $4.00 \pm 0.41$ & $1.89 \pm 0.13$ & $40.26 \pm 0.98$ \\
\hline & 5.4 & $85.0 \pm 2.9$ & $2.75 \pm 0.25$ & $1.74 \pm 0.06$ & $35.77 \pm 2.87$ \\
\hline \multirow[t]{4}{*}{ 'Pristine' $\times$ 'Peppermint' } & 4.2 & $95.0 \pm 2.9$ & $3.00 \pm 0.41$ & $1.35 \pm 0.21$ & $45.95 \pm 6.29$ \\
\hline & 4.6 & $100.0 \pm 0.0$ & $2.50 \pm 0.50$ & $1.53 \pm 0.08$ & $49.25 \pm 1.90$ \\
\hline & 5.0 & $90.0 \pm 4.1$ & $2.75 \pm 0.48$ & $1.37 \pm 0.13$ & $44.25 \pm 4.83$ \\
\hline & 5.4 & $87.5 \pm 4.8$ & $3.25 \pm 0.75$ & $1.25 \pm 0.06$ & $42.42 \pm 3.28$ \\
\hline
\end{tabular}

${ }^{\mathrm{z}}$ Data are present as mean of four replications \pm SE. Data were statistically analyzed and were not significantly different at $P<0.05$.

${ }^{\mathrm{y}}$ Seed germination was recorded weekly and T100 (indicating germination speed) was calculated as the number of weeks reaching $100 \%$ of total germination. $\mathrm{MAP}=$ months after pollination. 


\section{Literature Cited}

Alt, D.S., J.W. Doyle, and A. Malladi. 2017. Nitrogen-source preference in blueberry ( $\mathrm{Vac}$ cinium sp.): Enhanced shoot nitrogen assimilation in response to direct supply of nitrate. J. Plant Physiol. 216:79-87.

Anderson, W.C. 1980. Tissue culture propagation of red and black raspberries, Rubus idaeus and R. occidentalis. Acta Hort. 112:13-20.

Askari-Khorasgani, O., F. Mortazaeinezhad, M. Otroshy, and A.R. Golparvar. 2013. Breaking seed dormancy of endangered medicinal plant Kelussia odoratissima using zygotic embryo culture technique. Tech. J. Eng. Appl. Sci. 3:1712-1718.

Bridgen, M.P. 1994. A review of plant embryo culture. HortScience 29:1243-1246.

Clark, M.B., H.A. Mills, C.D. Robacker, and J.G. Latimer. 2003. Influence of nitrate: Ammonium ratios on growth and elemental concentration in two azalea cultivars. J. Plant Nutr. 26:2503-2520.

De Jeu, M. 2000. In vitro techniques for ornamental breeding. Acta Hort. 508:55-60.

Dirr, M.A. 2011. Manual of woody landscape plants. 6th ed. Stipes Publishing, Champaign, IL.

Eeckhaut, T., E. De Keyser, J. Van Huylenbroeck, J. De Riek, and E. Van Bockstaele. 2007. Application of embryo rescue after interspecific crosses in the genus Rhododendron. Plant Cell Tissue Organ Cult. 89:29-35.

Gamborg, O.L., R.A. Miller, and K. Ojima. 1968. Nutrient requirements of suspension cultures of soybean root cells. Expt. Cell Res. 50:151-158.
Geng, X., Y. Wu, and H. Zhao. 2014. Preliminary study on hybrid embryo rescue of Rhododendrons. J. Yunnan Agr. Univ. 29:533-539.

Hao, Y., H. Wang, R. Yang, F.L. Wang, and J. Lu. 2014. Effectiveness of culture media on the success of embryo rescue among different crosses of seedless grapes. Acta Hort. 1046:259-263.

Jaynes, R.A. 1971. Seed germination of six Kalmia species. J. Amer. Soc. Hort. Sci. 96:668-672.

Jaynes, R.A. 1982. New mountain laurel selections and their propagation. Intl. Plant Prop. Soc. Proc. 32:431-434.

Jaynes, R.A. 1988. Kalmia: The laurel book. Timber Press, Portland, OR.

Lloyd, G. and B. McCown. 1980. Commerciallyfeasible micropropagation of mountain laurel, Kalmia latifolia, by use of shoot-up culture. Intl. Plant Prop. Soc. Proc. 30:421-427.

Malek, A.A., F.A. Blazich, S.L. Warren, and J.E. Shelton. 1989. Influence of light and temperature on seed germination of mountain laurel. J. Environ. Hort. 7:161-162.

McCown, B.H. 2000. Recalcitrance of woody and herbaceous perennials plants: Dealing with genetic predeterminism. In Vitro Cell. Dev. Biol. Plant 36:149-154.

Michishita, A., K. Ureshino, and I. Miyajima. 2001. Shortening the period from crossing to the seedling stage through ovule culture of interspecific crosses of azalea (Rhododendron spp.). J. Jpn. Soc. Hort. Sci. 70:54-59.

Murashige, T. and F. Skoog. 1962. A revised medium for rapid growth and bioassays with tobacco tissue cultures. Physiol. Plant. 15:473-497.
Pavlovic, S., B. Vinterhalter, S. Zdravkovic-Korac, D. Vinterhalter, J. Zdravkovic, D. Cvikic, and N. Mitic. 2012. Recurrent somatic embryogenesis and plant regeneration from immature zygotic embryos of cabbage (Brassica oleracea var. capitata) and cauliflower (Brassica oleracea var. botrytis). Plant Cell Tissue Organ Cult. 113:397-406.

Reeves, D.W., B.D. Horton, and C.A. Couvillon. 1983. Effect of media and media $\mathrm{pH}$ on in vitro propagation of 'Nemaguard' peach rootstock. Scientia Hort. 21:353-357.

Ribeiro, V.G., M. Psaqual, J.D. Ramos, G.R. Carvalho, and A.F. Oliveira, Jr. 1999. Effect of agar and $\mathrm{pH}$ on in vitro orange 'Natal' cultivar embryo. Rev. Ceres 46:587-595.

Schneider, C.A., W.S. Rasband, and K.W. Eliceiri. 2012. NIH Image to ImageJ: 25 years of image analysis. Nat. Methods 9:671-675.

Sharma, D.R., R. Kaur, and K. Kumar. 1996. Embryo rescue in plants-A review. Euphytica 89:325-337.

Sharma, H.C. 1995. How wide can a wide cross be? Euphytica 82:43-64.

Taiz, L. and E. Zeiger. 2010. Plant physiology. 5th ed. Sinauer Assoc., Sunderland, MA.

Yang, Y., D. Zhang, Z. Li, X. Jin, and J. Dong. 2015. Immature embryo germination and its micropropagation of Ilex crenata Thunb. HortScience 50:733-737.

Zhang, M., M. Han, X. Wang, Y. Tian, S. Wang, A. Wang, and X. Zhu. 2004. Factors affecting embryo rescue of early ripening nectarines. J. Fruit Sci. 21:382-384. 